\title{
Incidence of the Curvature of a Catheter on the Variations of the Inner Volume: Application to the Peripherally Central Catheters
}

\author{
Gérard Guiffant, ${ }^{1}$ Patrice Flaud, ${ }^{1}$ Philippe Dantan, ${ }^{1}$ \\ Christian Dupont, ${ }^{2}$ and Jacques Merckx ${ }^{1,3}$ \\ ${ }^{1}$ Matière et Systèmes Complexes, UMR CNRS 7057, University Paris Diderot, 75205 Paris Cedex 13, France \\ ${ }^{2}$ Cystic Fibrosis Center, Hôpital Cochin, Pneumology, AP-HP, 75679 Paris Cedex 14, France \\ ${ }^{3}$ Anesthesia Department, University Teaching Hospital Necker-Enfants Malades, 75743 Paris Cedex 15, France
}

Correspondence should be addressed to Gérard Guiffant, gerard.guiffant@univ-paris-diderot.fr

Received 20 September 2012; Accepted 22 October 2012

Academic Editors: D. Sander and A. Suzuki

Copyright (C) 2012 Gérard Guiffant et al. This is an open access article distributed under the Creative Commons Attribution License, which permits unrestricted use, distribution, and reproduction in any medium, provided the original work is properly cited.

\begin{abstract}
One particular characteristic of peripherally central catheter (PCC), peripherally inserted central catheter (PICC) or totally implantable venous access device (TIVAD), is to be submitted to a rotational movement around the shoulder in the daily routine. The aim of this paper is to show that these movements induce a variation of the inner volume of the catheter which may be responsible for an internal distal pollution of the lumen. The results are presented in the framework of an open discussion on the catheter protocols of use as well as a possible improvement of the materials.
\end{abstract}

\section{Introduction}

The use of vascular access devices has become a standard practice [1-8], so that it is impossible to mention all the situations where a catheter is used. A catheter can be roughly described as a cylindrical flexible duct made of polymer (silicone or polyurethane), permitting either blood collection or injections, namely, drug solutes, lipids, contrast agents, and so forth. It is well known that one of the main difficulties in catheter management is to ensure the patency of the lumen by frequent and effective flushing. The causes of internal pollution are numerous and complex and are the result of a series of physicochemical processes.

Among all the possible causes, it is questionable to examine the possible mechanical effect of the variation of the curvature on the inner volume of the catheter. Such an effect can occur, for example, when a catheter is inserted in a vein and too long, as it is sometimes the case, rolled up on the forearm. During a change of bandage or any manipulation, the catheter is unrolled, and its internal volume is therefore modified. A second possibility can occur when a catheter inserted in the arm or forearm [9], namely, peripherally central catheter (PCC) either peripherally inserted central catheter (PICC) or totally implantable venous access device (TIVAD), is submitted to a rotational movement around the shoulder in the movements of everyday life. These effects will be described with more details in the following. The reasons for such behaviour must be searched in the mechanical properties of the materials constituting the catheters. As a flexible material, the behaviour of the catheter is dependent on two mechanical parameters: the Young modulus which is representative of the elasticity and the Poisson's ratio coefficient representative of the material contraction when submitted to a longitudinal stretch.

The first aim of this paper is to report some experimental and practical observations leading to the conclusion that any change in the curvature of a catheter, or of the infusion line, can be related to a change in the inner volume. Such an effect may induce a suction effect at the distal extremity of the catheter, thus leading in fine to an internal, permanent, and repeated pollution of the wall of the catheter. This type of processes could be responsible for the distal pollution 
observed after a catheter removal. We report some numerical results on the effect of the curvature of a catheter on the internal volume.

The second aim is to investigate the impact of both the geometrical and mechanical parameters on the variations of the inner volume of the catheters when bent. The results are presented for different parameters, namely, nature of the material, internal diameter, and thickness of the wall, thus leading to a possible practical recommendation on the catheters implementation.

\section{Experimental and Practical Observations}

The manipulation of a flexible catheter (PCC) is naturally accompanied with curvature changes. The effect on the internal volume can be easily measured by tracking the interface of a coloured liquid inside of a catheter closed at one extremity.

Figure 1 shows the displacement of the coloured interface obtained with a catheter of $0,5 \mathrm{~mm}$ internal diameter as a function of the curvature radius for a complete loop of the catheter. We observe that the displacement (consequently, the variation of the inner volume) is even more important as the radius is small. For increasing values of the curvature radius (the catheter tends to be straight), the displacement becomes negligible. It is to be noted that the results indicate that the internal volume of the catheter is greater when the catheter is straight. In other words, rolling the catheter induces an effect of reflux from the catheter, while unrolling induces a suction effect.

A practical situation of variation of the curvature of a catheter can be found when observing the behaviour of an installed PCC. Figure 2(a) shows schematically the path of a PCC. The implantation of a brachial or forearm inserted central catheter (PCC) requires a semicircular trajectory of the catheter from the forearm to its end in the vena cava, above the right atrium. It describes a wide curve of $30-40 \mathrm{~cm}$ long for which only the outer segment is movable with the upper limb. The internal segment (axillary vein, subclavian vein, and vena cava) has "low mobility" in the chest cavity.

The venous drainage (cf. Figures 2(b), 2(c), and 2(d)) of the upper limb is provided by the subclavian vein arose from the axillary vein, medial brachial vein(s) extension (3). Basilic vein (2) joins a vein of deep network, the medial brachial and sometimes axillary vein. The cephalic vein (1) joins, with a curve, the axillary vein. Basilic and cephalic veins empty the superficial network.

Figures 2(b), 2(c), and 2(d), extracted from phlebography (courtesy of Dr. Boudjemline, Necker EM, Pr. Sidi., Paris), show the anatomical modifications of the veins of the upper limb, for different positions of this limb: alongside the body (b), $90^{\circ}$ abduction (c), and incomplete verticalisation (d). The axis of rotation of the venous network and thus of the inserted PCC is then the centre of the head of the humerus. In the daily life, these movements, occurring in a frontal plane as described above, are combined with movements in the sagittal plane (ante or retropulsion). Thus, varied and combined joint motion of the scapulohumeral

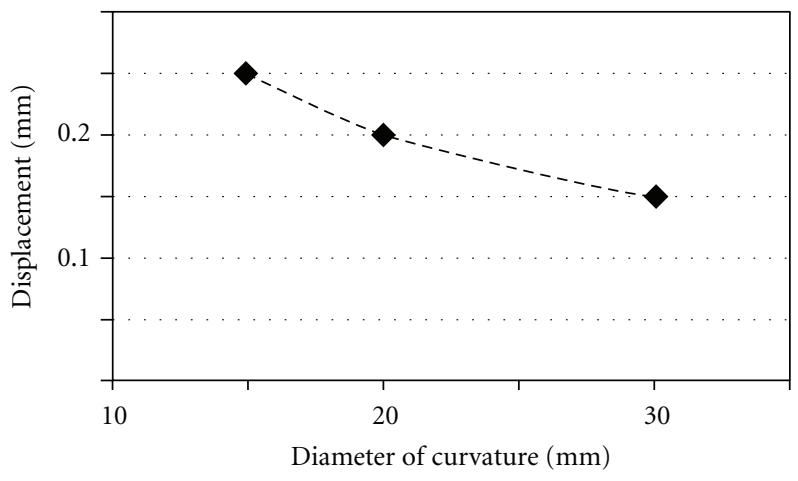

FIGURE 1: Experimental recording of the displacement of the coloured liquid interface obtained with a catheter of $0,5 \mathrm{~mm}$ internal diameter as a function of the curvature radius for a complete loop of the catheter.

joint involves geometrical changes in the venous network and therefore of the inserted PCC. In the movements of everyday life, the position of the upper limb has a large amplitude, $0^{\circ}$ to $90^{\circ}$ or even more, near $180^{\circ}$, in abduction, antepulsion, and verticalization (cf. Figures 2(b), 2(c), and $2(d))$. It is clearly difficult to assess the exact magnitude of the venous path around the scapulohumeral joint, during these movements. The radius of the head of humerus is about $24 \mathrm{~mm}$, then a rough estimation of the catheter curvature leads to a radius of approximately $27-30 \mathrm{~mm}$.

Figure 3 shows a typical magnified picture of the extremity of a PICC after being removed. One can see a small internal polluted area around the extremity of the catheter. This observation is seen as an index of the possible relationship between the internal distal pollution of the inserted catheter and different variation of the curvature of installed catheters associated with variations in internal volume. These volume changes induce a "suction" of blood and then a catheter distal pollution. This pollution may extend iteratively and gradually fill the extremity of the catheter. It can also serve as the attachment point of a thrombus of fibrin and platelets.

\section{Numerical Model and Results of the Simulations}

A numerical approach (COMSOL) has been performed for studying the relationship between the internal volume of a catheter and the curvature. Such an approach permits to test the influence of each geometrical and physical parameter. The material of the catheter (silicone or polyurethane) is characterized by two parameters: namely, the Young modulus $E$ describing the elastic properties of the material, with $E$ ranging in the interval from 0,1 MegaPascals to 10 MegaPascals for these materials, and Poisson's ratio $v$ is representative of the ability of the material to contract when stretched. Because of its definition, the numerical values of this nondimensional coefficient are strictly positive and comprised between 0 and 0,5 [10]. The catheter is chosen to be $20 \mathrm{~cm}$ long with three internal diameters $d: 2 \mathrm{~mm}$, 


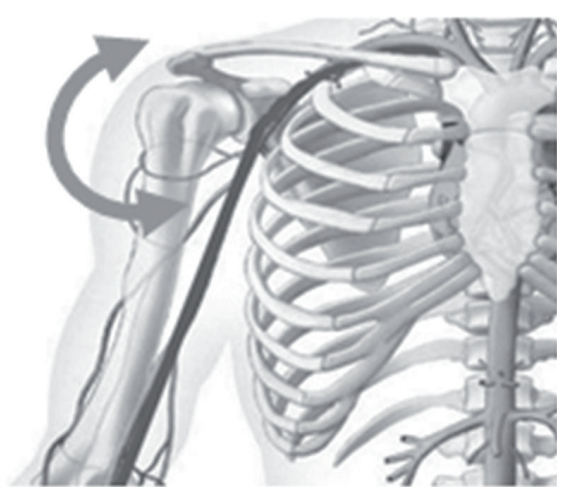

(a)

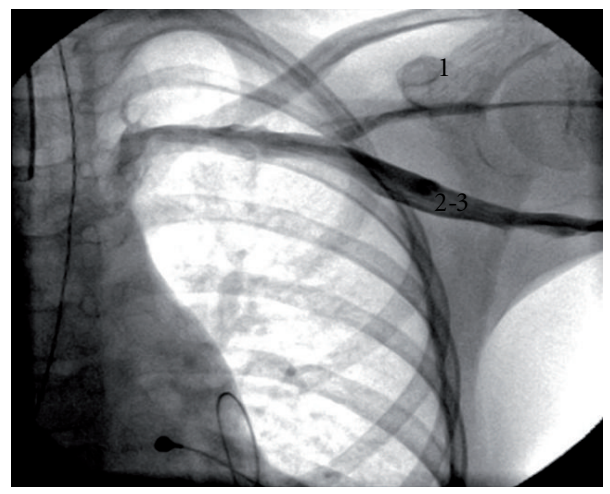

(c)

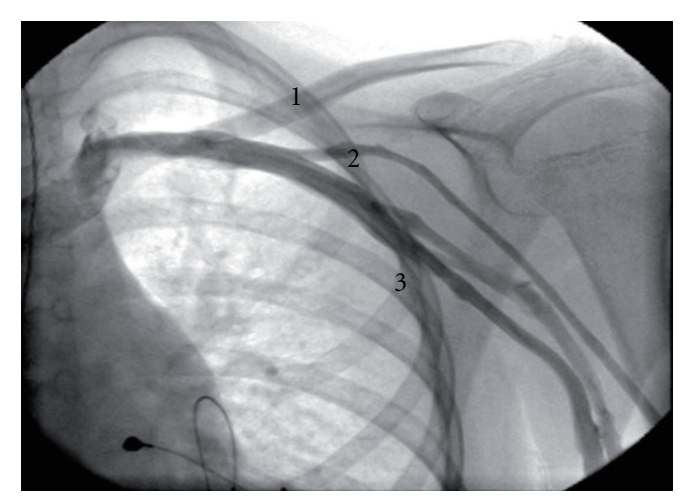

(b)

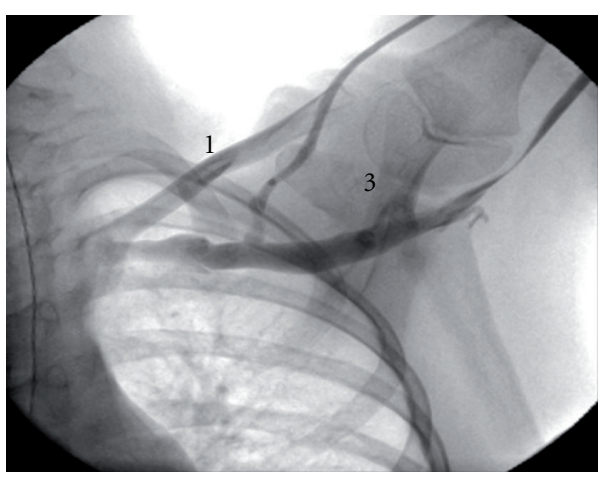

(d)

FIGURE 2: 2(a) Schematic representation of the path of a PCC, 2(b), (c), and (d) phlebography, showing the anatomical modifications of the veins of the upper limb, for different positions: alongside the body (b), $90^{\circ}$ abduction (c), and incomplete upright movement (d).

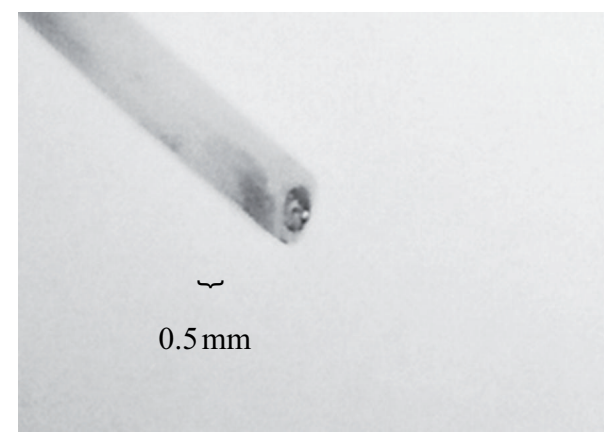

FIGURE 3: Typical magnified picture of the extremity of a PICC after being removed.

$3 \mathrm{~mm}$, and $4 \mathrm{~mm}$. For each internal diameter, three possible thicknesses have been tested. All the simulations have been performed under the same catheter deformation.

Figure 4 shows the deformation applied to the catheter. One extremity of the catheter is rotated in such a way that the axis of the catheter forms an angle of $45^{\circ}$ with the initial direction of the catheter, while the other extremity is maintained fixed. The numerical scheme permits to express the percentage of internal volume variation of the catheter resulting from the curvature. The simulations have been performed for different mechanical parameters (Young modulus and Poisson's ratio), varying the geometrical characteristics.

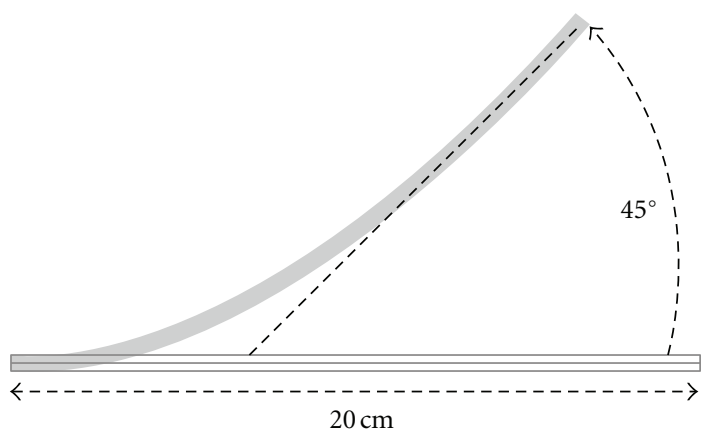

FIGURE 4: Geometrical deformation imposed to the catheter for the numerical simulation.

The first significant result is that no effect has been found when varying the Young modulus for the different geometrical parameters selected. In other words, the elasticity of the catheters does not influence the internal volume when bent.

Figures 5(a), 5(b), and 5(c) and Table 1 show the results obtained when varying Poisson's ratio for different values of the geometrical parameters. The results are presented in the same scales of variation to facilitate the comparisons. As mentioned above, Poisson's ratio is restricted to the interval $0-0.5$. The variations of internal volume are given in the percentage of variation with respect to the initial value 


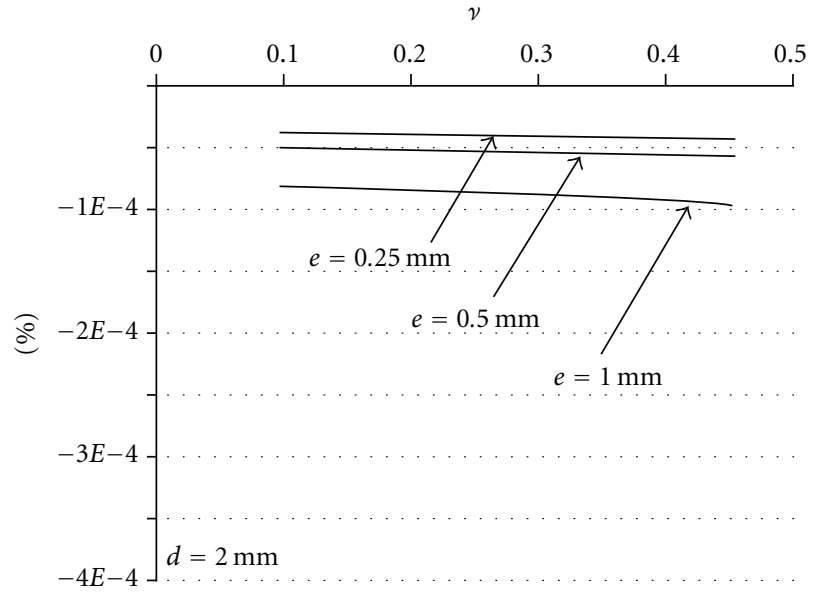

(a)

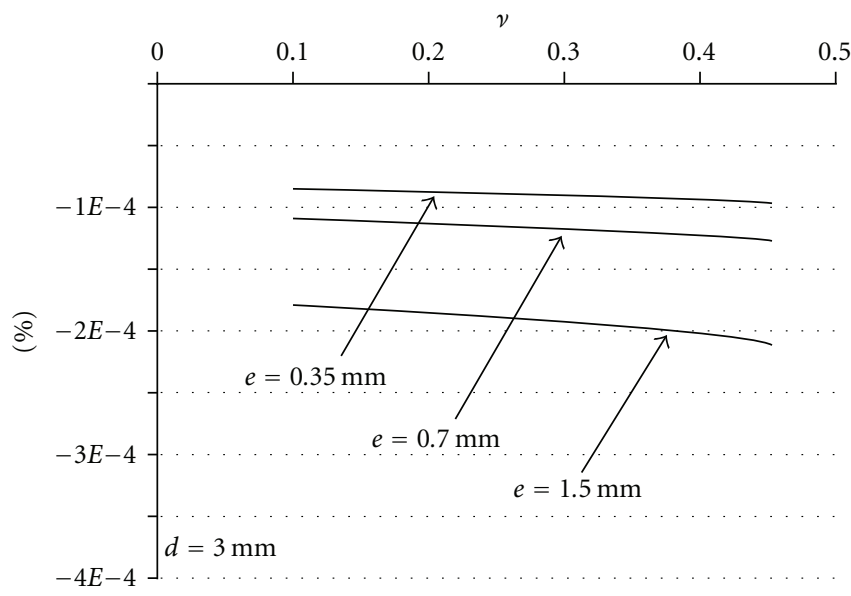

(b)

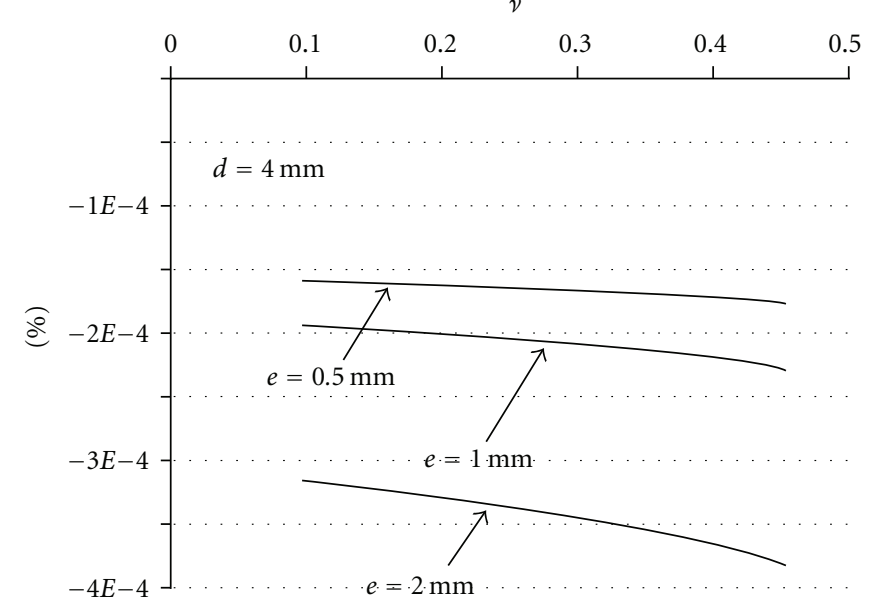

(c)

FIGURE 5: Numerical results giving the percentage of variation of the inner volume as a function of the coefficient of Poisson's ratio for different values of the inner diameter $d$ and for different values of the thickness $e$ of the catheter.

TABLE 1: Numerical values of the percentage of variation of the inner volume for different values of the inner diameter $d$ and for different values of the thickness $e$ of the catheter, obtained from the numerical simulation for the maximum possible value of the Poisson's ratio $v=0,5$.

\begin{tabular}{lccc}
\hline \multirow{2}{*}{$d=2 \mathrm{~mm}$} & $e=0,25 \mathrm{~mm}$ & $e=0,5 \mathrm{~mm}$ & $e=1 \mathrm{~mm}$ \\
& $-4,25 E-05$ & $-5,68 E-05$ & $-9,81 E-05$ \\
& $e=0,35 \mathrm{~mm}$ & $e=0,7 \mathrm{~mm}$ & $e=1,5 \mathrm{~mm}$ \\
$d=3 \mathrm{~mm}$ & $-9,85 E-05$ & $-0,00013$ & $-0,00022$ \\
& $e=0,5 \mathrm{~mm}$ & $e=1 \mathrm{~mm}$ & $e=2 \mathrm{~mm}$ \\
\multirow{2}{*}{$d=4 \mathrm{~mm}$} & $-0,00018$ & $-0,00024$ & $-0,00040$ \\
\hline
\end{tabular}

when the catheter is straight. The percentages of variation are negative because when the catheter is subjected to a curvature, the internal volume undergoes a diminution. Figures 5(a), 5(b), and 5(c) give the results for three internal diameters, namely, $d=2 \mathrm{~mm}, 3 \mathrm{~mm}$, and $4 \mathrm{~mm}$ in the nondeformed situation. For each initial internal diameter, three thicknesses, $e$, have been tested: $e=0.25 \mathrm{~mm}-$ $0.5 \mathrm{~mm}-1 \mathrm{~mm}$ for $d=2 \mathrm{~mm}$; $e=0.35 \mathrm{~mm}-0.7 \mathrm{~mm}-1.5 \mathrm{~mm}$ for $d=3 \mathrm{~mm}$, and $e=0.5 \mathrm{~mm}-1 \mathrm{~mm}-2 \mathrm{~mm}$ for $d=4 \mathrm{~mm}$. These different values are reported on the figures. In the framework of the present study, the main contribution of the numerical results is not the particular values of the percentages of variation of the internal volumes but rather the types of dependence as a function of Poisson's ratio. All the simulations show that the decisive mechanical parameter is the Poisson's ratio for the incidence of the curvature on the internal volume of a catheter. It appears that, for smaller values of both internal diameter and thickness, the impact of the curvature on the internal volume is less often, associated with the smaller values of the Poisson's ratio. It follows then from these results that it should be convenient to introduce these considerations among all the criteria which have to be considered for any catheter development. 


\section{Discussion}

The development of the PCC leads to the awareness of the importance of the obstruction which is the most common complication in $3 \%$ to $25 \%$ of the cases when using central vascular access as mentioned by Bartock [11]: "PICC occlusions are a highly prevalent clinical concern that has many patient, family, community and health care system implications. The clinical and economical costs are great, and reduction of occlusion rates can significantly improve the quality of healthcare for many patients." Different processes of obstruction are discussed [12]: biological, mechanical, and physical, but the causes are rarely unique although frequently from a thrombolytic origin $[1,13]$. Most of the time, the pollution is the result of a succession of deposits on the endoluminal sheath protein: quick deposit after setting up the equipment, deposit of proteins from blood or therapeutic origin, platelets or red blood cells, and physical or chemical precipitates from pharmacological origin.

The comparison between different publications is made difficult because of the heterogeneity of the populations, pathologies, and therapies. The physical and geometrical characteristics of the PICCs are diverse; material and particularly internal diameter are not taken into account. For example, for the same external diameter, the internal diameter of a polyurethane catheter is higher than the internal diameter of a silicone catheter. In addition, the diameters of the paediatric catheters are lower than the adult catheter which is probably the cause of the greater number of occurrences of obstruction found among children [13]. The diameter of the catheters is rarely noted in the literature [12]. The classical diameter of a CVC was and is still 2.1$2.6 \mathrm{~mm}(14-12 \mathrm{G}$ or $7-8 \mathrm{~F})$ [12] for the adult while the current diameter of a PICC is $1.3-1.6 \mathrm{~mm}(18-16 \mathrm{G}$ or $4-5 \mathrm{~F})$ and recently $0.57 \mathrm{~mm}$ or $1.9 \mathrm{~F}$. The extent of the pollution or obstruction is never similar from one catheter to another. It is only appreciable after the removal if the catheter is cut out or is transparent which is rarely the case, the catheters being most often opaque. In fact, one consequence of all these disparities is the multiplicity of the desobstruction processes [14]. The activity of the patient is never reported, and the increase of the hospitalisation at home is associated with an increase in mobility and activity of the patients which remains higher than in case of hospitalization. To our knowledge, the possible variations of the internal volume of the PICCs have never been described in the literature. The impacts on the vacuity of the lumen of the vascular accesses are then less dependent on the internal volume itself than to the repetition and summation of the effects induced by the variations of curvature.

Two questions appear to be essential:

(i) why a fibrin hematic deposit can be often observed after the removal of a PCC even when well maintained?

(ii) is this deposit important enough to have repercussions on the emptiness of the PCC and infectious consequences?
The intraluminal backflow "pump effect" occurs as soon as the relevant member is moving. The volume of reflux is small and even null when the PCC is perfused, but when out of perfusion, the small volumes of reflux are cumulative. This accumulation can locally induce a decrease of the lumen and become then the point of attachment of a septic settlement. The practical and clinical consequences are obviously difficult to evaluate. The protocols of maintenance of the PCC in intertreatment recommend more frequent flushing than for other central catheters. Then, it should be convenient to achieve an evaluation of the level of the terminal pollution and obstruction (or infection) of the PCC as a function of the frequency of flushing.

It is an evidence that the achievement of a catheter intended for use as PCC results of a compromise between a variety of constraints including biochemical criteria (biocompatible polymer) and mechanical criteria (resistance and flexibility). Among all the constraints, we suggest to take into account the necessity of choosing the geometrical characteristics and the mechanical properties of the material so as to reduce the variation of internal volume when the catheter is submitted to bending. The silicone and polyurethane commonly used have mechanical properties which are widely dependent on the composition of the polymers. Some papers [15-17] give typical values of the Poisson's ratio. For the polyurethane the value of $v$ is commonly found in the intervals 0.39 to 0.499 while for the silicone, the values of $v$ lie in the interval from 0.22 to 0.34 depending on the process used for obtaining the polymer. It is thought that taking into account this approach should contribute to reduce the possible occurrences of distal pollution.

\section{Conclusions}

Following the values of $v$ from the literature, the silicone appears as a better candidate for reducing the effect of variation of internal volume although the gap is small for small internal diameter and small thickness (cf. Figure 5(a)). An alternative approach would be to preserve the use of polyurethane because of its properties of mechanical resistance but with a special attention both to the composition and the process of synthesis permitting thickness and Poisson's ratio as small as possible. Although the best prevention for the inner pollution remains frequent and effective [18], flushing it may be useful to recommend to the patient to avoid significant movements of the arm leading to a pump effect as described above. Moreover, from a global point of view, it is suggested that it should be convenient to take into account the mechanical processes reported in this paper for the definition of protocols including the manipulation of catheters in order to prevent any distal pollution which may result from mechanical manipulations.

It is well known that the pathogenesis of this pollution is multifactorial $[19,20]$, but endothelial injury, turbulence of the venous flow, and catheter thrombogenicity play a role, as does the composition of the infusate and the characteristics of the disease process. It is simply suggested here that among 
all these factors the change in the curvature of a catheter leading to a suction effect at the distal extremity would be responsible for distal pollution. It could be then reasonable to reduce such reflux by the use of devices where the catheter tip is valved. The question remains an open question because the flow in the catheter in the vicinity of the valve (distal or proximal) can generates the so-called stagnation zone which may promote an obstructive, infectious, and thrombotic risk.

\section{Conflict of Interests}

The authors reported no conflict of interests in this work.

\section{Acknowledgments}

The authors thank Dr. Boudjemline and Professor Sidi, Pediatric Cardiology in Necker Enfants Malades Hospital, Paris for supplying some records of phlebography.

\section{References}

[1] S. L. Herbst, "Options for venous access in ambulatory care: issues in selection and management," The Journal of Infusional Chemotherapy, vol. 6, no. 4, pp. 186-194, 1996.

[2] J. R. Hoch, "Management of the complications of long-term venous access," Seminars in Vascular Surgery, vol. 10, no. 3, pp. 135-143, 1997.

[3] M. Driscoll, C. Buckenmyer, M. Spirk, and C. Molchany, "Inserting and maintaining peripherally inserted central catheters," Medsurg Nursing, vol. 6, no. 6, pp. 350-358, 1997.

[4] J. Todd, "Peripherally inserted central catheters and their use in IV therapy," British Journal of Nursing, vol. 8, no. 3, pp. 140146, 1999.

[5] L. Chase, R. Nicol, B. Ferreira et al., "Evolution of a PICC program: a Canadian perspective," in Proceedings of the 18th Annual AVA Conference, Vancouver, Canada, 2004.

[6] J. Pettit, "Technological advances for PICC placement and management," Advances in Neonatal Care, vol. 7, no. 3, pp. 122-131, 2007.

[7] V. Vidal, A. Jacquier, O. Monnet et al., "Peripherally inserted central catheters (PICC): a promising technique," Journal de Radiologie, vol. 89, no. 7-8, pp. 907-909, 2008.

[8] V. Vidal, C. Muller, A. Jacquier et al., "Prospective evaluation of PICC line related complications," Journal de Radiologie, vol. 89, no. 4, pp. 495-498, 2008.

[9] D. F. Denny, "Placement and management of long-term central venous access catheters and ports," American Journal of Roentgenology, vol. 161, no. 2, pp. 385-393, 1993.

[10] Y. C. Fung, Biomechanics, Mechanical Properties of Living Tissues, Springer, New York, NY, USA, 1981.

[11] L. Bartock, "An evidence-based systematic review of literature for the reduction of PICC line occlusions," Journal of the Association for Vascular Access, vol. 15, no. 2, pp. 58-63, 2010.

[12] M. E. Juvé, "Intravenous catheter declotting: same outcomes with lower dose urokinase?" Journal of Infusion Nursing, vol. 26, no. 4, pp. 245-251, 2003.

[13] C. Tolomeo and W. Mackey, "Peripherally inserted central catheters (PICCs) in the CF population: one center's experience," Pediatric Nursing, vol. 29, no. 3, pp. 355-359, 2003.

[14] Ch. Dupont and P. R. Burgel, "Peripherally-inserted central catheter for intravenous antibiotics in adult patients with cystic fibrosis orbronchiectasis," "European Cystic Fibrosis Congress. In press.

[15] C. A. Harper, Handbook of Plastics, Elastomers \& Composites, McGraw-Hill, New York, NY, USA, 2002.

[16] M. A. Hopcroft, W. D. Nix, and T. W. Kenny, "What is the Young's modulus of silicon?" Journal of Microelectromechanical Systems, vol. 19, no. 2, pp. 229-238, 2010.

[17] V. G. Sazonov, "Rigidity of polyurethane in various loading conditions," Russian Engineering Research, vol. 29, no. 6, pp. 563-570, 2009.

[18] G. Guiffant, J. J. Durussel, J. Merckx et al., "Flushing of intravascular access devices (IVADs) Efficacy of pulsed and continuous infusions," Journal of Vascular Access, vol. 13, no. 1, pp. 75-78, 2012.

[19] N. P. O’Grady, M. Alexander, E. P. Dellinger et al., "Guidelines for the prevention of intravascular catheter-related infections. Centers for Disease Control and Prevention," MMWR, Recommendations and Reports, vol. 51, no. 10, pp. 1-29, 2002.

[20] N. P. O'Grady, M. Alexander, L. A. Burns et al., "Guidelines for the prevention of intravascular catheter-related infections," Clinical Infectious Diseases, vol. 52, no. 9, pp. 1087-1099, 2011. 


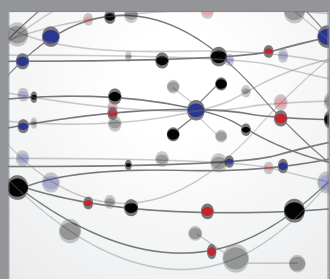

The Scientific World Journal
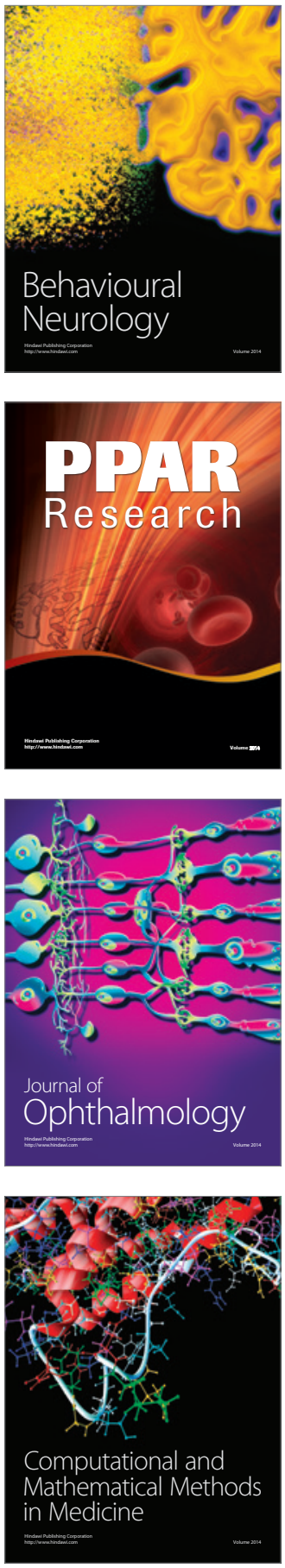

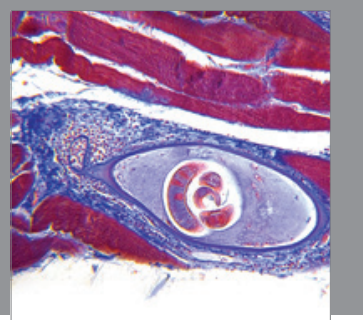

Gastroenterology

Research and Practice
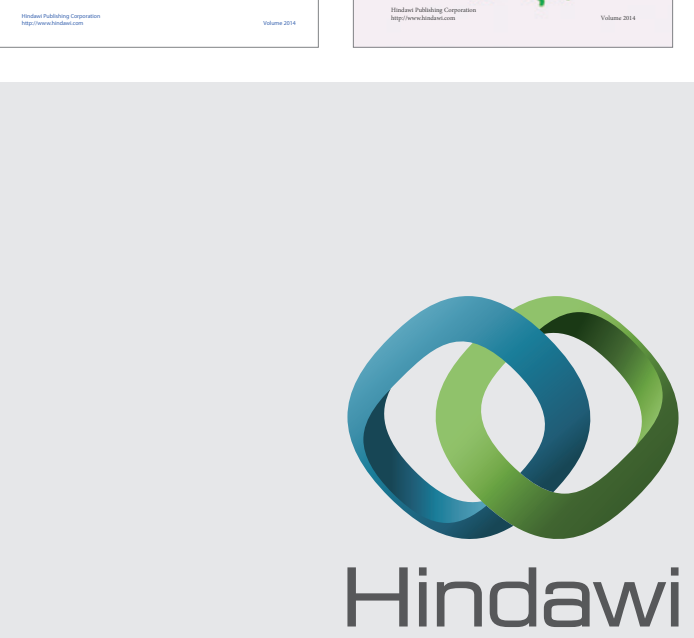

Submit your manuscripts at

http://www.hindawi.com
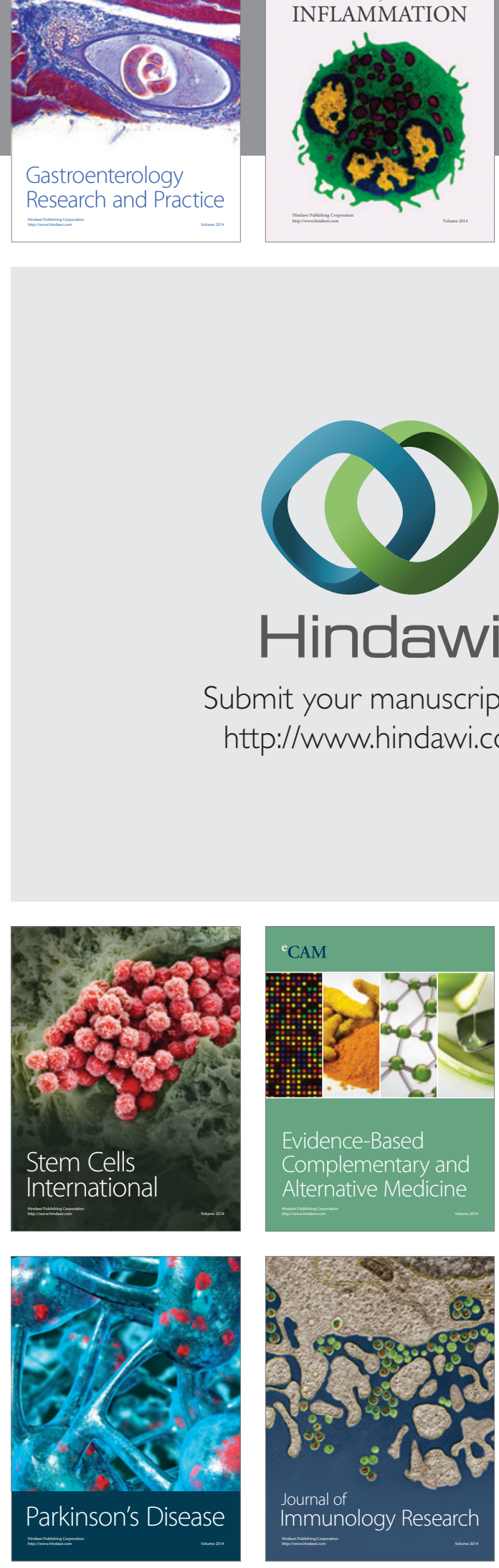

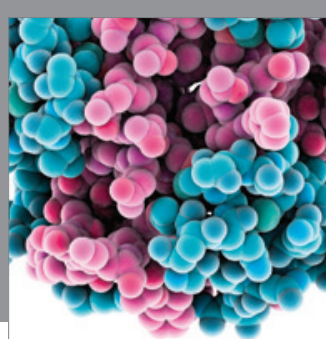

Diabetes Research
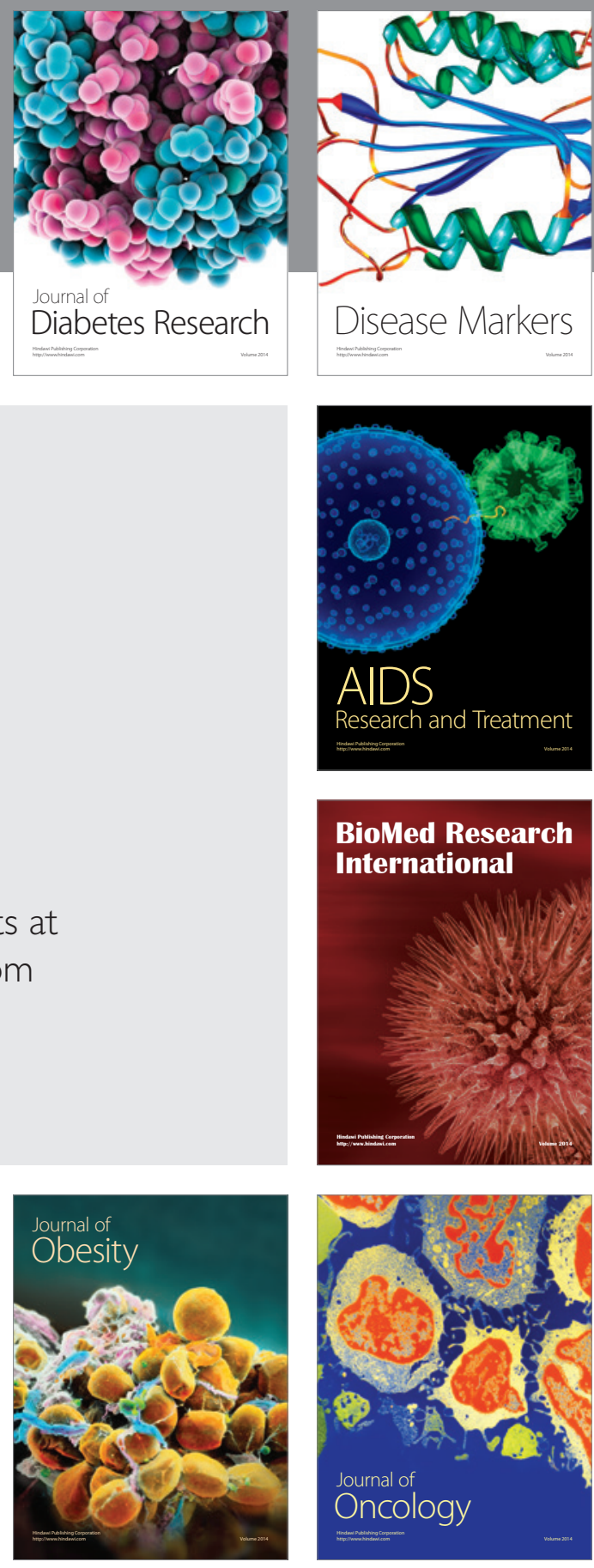

Disease Markers

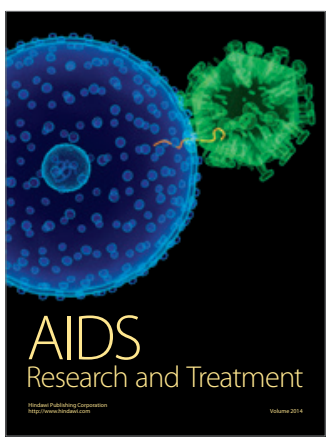

BioMed Research

International
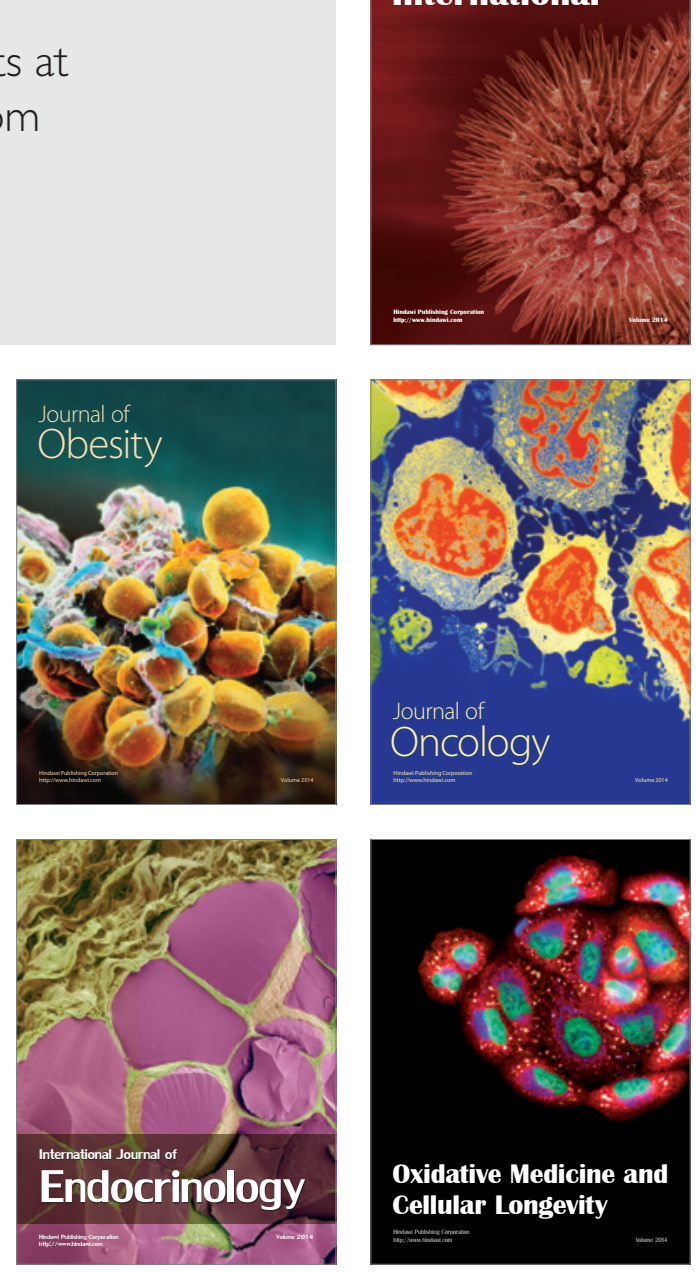